\title{
Redes queer: escritoras, artistas y mecenas en la primera mitad del siglo $X X$
}

Queer networks: writers, artists, and sponsors in the first half of the 20th century Redes queer: escritoras, artistas e patronos na primeira metade do século XX

\section{Claudia Cabello Hutt}

UNIVERSITY OF NORTH CAROLINA, ESTADOS UNIDOS

Profesora asociada y directora de posgrado en el Departamento de Lenguas, Literaturas y Culturas de la Universidad de North Carolina, Greensboro. Es doctora en Literatura Latinoamericana con una concentración en Estudios de Género de la Universidad de Rutgers. Su investigación se enfoca en la modernidad latinoamericana, la historia intelectual, teoría de redes y estudios de género. Es autora de Artesana de sí misma: Gabriela Mistral, una intelectual en cuerpo y palabra (Purdue UP, 2018). Su proyecto actual se enfoca en redes queer en el campo cultural entre 1920 y 1950. Correo electrónico: c_cabell@uncg.edu 


\section{Resumen}

A partir de la lectura del epistolario de Anna Melissa Graves, este trabajo recompone la relación de complicidad entre la escultora chilena Laura Rodig y la, hasta ahora desconocida "mecenas", Consuelo Lemetayer. De manera específica, esta indagación de archivo analiza las redes queer de cooperación transnacional y transatlántica en las que participan estas intelectuales y artistas de la primera mitad del siglo XX. De manera general, el análisis contribuye a la reconstrucción, cuando menos parcial, de una historia cultural de mujeres escamoteadas por las memorias nacionales hegemónicas.

Palabras clave: queer; mujeres latinoamericanas (siglo XX); Laura Rodig; Consuelo Lemetayer; Anna Melissa Graves

\section{Abstract}

Based on the reading of the correspondence of Anna Melissa Graves, this work resbuilds the complicity relationship between the Chilean sculptor Laura Rodig and the, until now unknown "sponsor," Consuelo Lemetayer. Particularly, this archive inquiry analyzes the queer networks of transnational and transatlantic cooperation in which these intellectuals and artists of the first half of the 20th century took part. In a general way, the analysis contributes to the (at least partial) reconstruction of a cultural history of women scamped by hegemonic national memories.

Keywords: queerness; Latin American women (20th century); Laura Rodig; Consuelo Lemetayer; Anna Melissa Graves

\section{Resumo}

A partir da leitura do epistolário de Anna Melissa Graves, este trabalho recompõe o relacionamento de cumplicidade entre a escultora chilena Laura Rodig e a, até então desconhecida "patrona", Consuelo Lemetayer. De maneira especifica, esta indagação de arquivo analisa as redes queer de cooperação transnacional e transatlântica nas que essas intelectuais e artistas da primeira metade do século XX participam. De maneira geral, a análise contribui à reconstrução, quando menos parcialmente, de uma história cultural de mulheres escamoteadas pelas memórias nacionais hegemônicas.

Palavras-chave: queer; mulheres latino-americanas (século XX); Laura Rodig; Consuelo Lemetayer; Anna Melissa Graves

RECIBIDO: 15 DE JULIO DE 2016. ACEPTADO: 14 DE OCTUBRE DE 2016. DISPONIBLE EN LÍNEA: 29 DE DICIEMBRE DE 2017

\section{Cómo citar este artículo:}

Cabello Hutt, Claudia. "Redes queer: escritoras, artistas y mecenas en la primera mitad del siglo XX". Cuadernos de Literatura 21.42 (2017):

145-160. https://doi.org/10.11144/Javeriana.cl21-42.rqea 
EXISTE MUY POCA información biográfica acerca de la escultora chilena Laura Rodig (1901-1972). La parte de su vida que está más documentada corresponde al periodo en que convive con Gabriela Mistral. Rodig conoce a Mistral en Los Andes, a los 14 años, y, más tarde, en 1918, es nombrada profesora de arte en el Liceo de Punta Arenas, al mismo tiempo que Mistral asume la dirección de esta escuela. Permanecen en Punta Arenas hasta 1920, año en que ambas se trasladan a Temuco donde Rodig sufre hostilidad de parte del profesorado, que cuestiona su contratación o, más bien, como sugiere Martin C. Taylor, su relación con Mistral (93). Viajan juntas a México en 1922 por invitación de Vasconcelos a Mistral y, luego, Rodig parte rumbo a Europa, en 1924, destino donde su rastro empieza a desaparecer. En las biografías oficiales solo se indica que Rodig viaja con una beca del Gobierno de Chile. Durante este periodo realiza importantes muestras, por ejemplo, en 1928, en el Salón de Otoño de París, año en que también gana la medalla de oro en la exposición de Sevilla; en 1930, participa en la exposición de un grupo de latinoamericanos en la galería Zak y en el Salón des Tuileries (1930, 1931), entre otros premios y honores. Nunca se casa ni tiene hijos. ${ }^{1}$

$*$

Fines de los años 20. Otra mujer chilena, nacida en Valdivia en 1891, vive en París de sus rentas y negocios, asiste a exposiciones, se escribe con intelectuales y artistas, entre ellos José Carlos Mariátegui, con quien colabora gestionando las subscripciones para la revista Amauta. En 1923 participa como delegada en el Congreso de la Federación Internacional de Mujeres Trabajadoras (IFWW) en Viena. ${ }^{2}$ Además de viajar, escribir cartas y cuidar a sus dos hijas, esta mujer apoya y gestiona la carrera artística de Laura Rodig, con quien vive en París entre 1927 y 1931. Su nombre es Consuelo Lemetayer. $\mathrm{Ni}$ ella ni sus corresponsales mencionan su estado civil ni tampoco hacen

1 Rodig pasa sus últimos años en Santiago de Chile, donde tiene un taller en la casona de la pintora Inés Puyó en la calle Monjitas. Según testimonio de Susana Peña Castro, quien junto a su pareja visitó a Rodig allí poco antes de su muerte, la escultora vivía con una mujer, quien parecía ser su pareja de años.

2 Consuelo Lemetayer aparece como delegada fraternal (no representante de un sindicato de trabajadores afiliado) en el boletín del Sindicato Nacional de Mujeres de América. Life and Labor Bulletin. Vol. II, Noviembre 1923. Su participación en el Congreso de Viena también es anunciada en la revista argentina Caras y Caretas en 1923 (Vol. 26). 
referencia al padre de sus hijas. ${ }^{3}$ Consuelo Lemetayer no figura en ningún libro ni artículo académico.

La estadounidense Anna Melissa Graves (1875-1964) estudió en la Universidad de Columbia, fue escritora, viajera y activista. Viajó por África, gran parte de Latinoamérica, China, Europa y el Medio Oriente. Visitó Perú por diez meses en 1922 y trabajó junto a feministas peruanas. Se desempeñó como maestra en varios países y publicó cuatro colecciones de correspondencia con personas de los lugares que había visitado. Dio charlas y escribió artículos a favor de la paz y en contra del racismo; fue miembro activo de la Liga Internacional de Mujeres por la Paz y la Libertad (WILPF). Nunca se casó. Fue amiga y mecenas del intelectual peruano Víctor Raúl Haya de la Torre. Mantuvo también una amistad a través de cartas con Gabriela Mistral desde el comienzo de la década de 1920 hasta los años $195^{\circ} .^{4}$

La investigación de archivos ha revelado, en la correspondencia, en fotografías y en la prensa, la historia de los vínculos de una serie de mujeres escritoras, artistas y gestoras culturales que vivieron sus vidas de modo queer; en oposición a lógicas heterosexuales y patriarcales de familia, sexualidad y reproducción. El punto de partida de esta investigación es el concepto de queerness según lo plantea Jack (Judith) Halberstam, quien se basa en la idea de modo de vida homosexual de Foucault, definido, con separación de la identidad sexual, como:

si tratamos de pensar acerca del queerness como el resultado de temporalidades extrañas, esquemas de vida imaginativos, y prácticas económicas

3 La presente investigación ha confirmado que Lemetayer estuvo casada con Carlos Grado, con quien tuvo dos hijas, Inés Elvira y Margot Grado Lemetayer, y de quien se separó alrededor de 1918. Luego de la separación, Consuelo sale de Chile rumbo a Estados Unidos junto a sus hijas. Desembarca en San Francisco, California, a fines de septiembre de 1918. Agradezco la confirmación de estos datos y el aporte de otros,justo antes de la publicación de este artículo, a Bernardita Mandiola, bisnieta de Lemetayer.

4 Para más información acerca de la relación entre Gabriela Mistral y Anna Melissa Graves, véase, Cabello Hutt, Claudia. "Tejiendo un sueño americano: el poder de las redes de Gabriela Mistral con los Estados Unidos en los años 1920-1930". 
excéntricas, separamos el queerness de la identidad sexual y nos acercamos al sentido del comentario de Foucault en "Friendship as a Way of Life" que "la homosexualidad amenaza a las personas en tanto "forma de vivir" más que como una forma de tener sexo" (310). En la formulación radical de Foucault, las amistades queer, las redes queer y la existencia de estas relaciones en el espacio y con relación al uso del tiempo trazan la particularidad y precisamente la amenaza percibida de la vida homosexual. (Halberstam 1; la traducción es mía) ${ }^{5}$

A partir de un concepto más amplio y complejo de queerness, en sus dimensiones temporales, de ritmos de vida y prácticas económicas, y de un trabajo de archivo - que presenta numerosas dificultades (sujetos errantes, falta de conservación de materiales y censura) - es posible configurar un mapa de mujeres latinoamericanas queer que operan durante la primera mitad del siglo XX. Mujeres que vivieron sus vidas creativas e intelectuales fuera de los parámetros tradicionales de matrimonio, reproducción y dependencia económica. Este trabajo solo aborda un pequeño fragmento de estas redes transnacionales y transatlánticas, mayormente ignoradas por la crítica y la historiografía patriarcal y heteronormativa que, al negar su sexualidad, sus modos alternativos de asociatividad, han malinterpretado a estas mujeres como solteras, solas, excéntricas o, incluso, como no existentes. Foucault en "Friendship as a Way of Life" habla específicamente de la homosexualidad masculina, sin embargo, su propuesta es aplicable, con ciertos matices, a las relaciones afectivas/eróticas entre mujeres. Foucault plantea que lo que hace perturbadora a la homosexualidad no es el contacto sexual homosexual, sino "el afecto, la ternura, la amistad, la fidelidad, la camaradería y el compañerismo" (Ethics 136), es decir, modos alternativos de relaciones interpersonales que operan en "la formación de líneas de fuerzas impredecibles" (136). En el caso de las relaciones amorosas y sexuales entre mujeres en el contexto latinoamericano ha predominado la tendencia a negarlas y enmascararlas. Esta negación, junto con prácticas violentas de normalización, ha afectado a escritoras como Gabriela Mistral y Teresa de la Parra, entre otras. En estos casos la idea de la amistad y la afectividad femenina se ha usado como un mecanismo de silenciamiento del potencial amenazador de estas relaciones al identificar a sus parejas como amigas, secretarias o compañeras de viajes.

5 Todas las traducciones de fuentes originalmente en inglés son mías a menos que se indique otra cosa. 
La crítica y las instituciones se han resistido, hasta hace muy poco, a pensar acerca de un cuerpo, un modo de vida y una asociatividad queer hasta que no se tenga prueba irrefutable de la relación sexual. Es posible sugerir, en línea con Foucault, que en el caso de las mujeres tampoco sería el acto sexual lo que más perturba al orden patriarcal, sino la resistencia frente a modelos de dominio del cuerpo, de reproducción y de control económico sobre la mujer. El concepto de queerness que, por su parte, propone Halberstam como producto de "temporalidades extrañas, esquemas de vida imaginativos y prácticas económicas excéntricas" (1) permite pensar en las líneas de fuerza que unen a estas mujeres, sus redes y los efectos de estos vínculos en sus carreras literarias, artísticas y en la historia cultural de Latinoamérica. Esta investigación se propone rescatar historias perdidas, censuradas y malinterpretadas, no solo por justicia histórica y como respuesta ante la violencia de los discursos normalizadores, sino como un medio de problematizar discursos hegemónicos en torno a la historia cultural e intelectual del siglo XX.

Volvemos entonces a la chilena Consuelo Lemetayer, su vida en París con la escultora Laura Rodig y las cartas que escribe a su amiga, Anna Melissa Graves. Este caso resulta fascinante dado que Lemetayer es un sujeto completamente desconocido para la crítica y la historiografía, que además tampoco aparece mencionada en los varios libros de testimonios y epistolarios de miembros de la comunidad chilena en París en los años 1920 y $1930 .{ }^{6}$ Lemetayer entra en escena gracias a un conjunto de veintiuna cartas inéditas pertenecientes al archivo de Anna Melissa Graves (Swarthmore College Peace Collection). Las cartas que esta mujer chilena le dirige a Graves van desde 1928 hasta 1931, pero sugieren que la relación de amistad se inicia antes. La mayoría de las cartas las escribe desde París, pero también desde Londres, Chile y Bruselas. Esta correspondencia remite a otros epistolarios que conectan a Graves con Mistral. A su vez, Mistral está vinculada con Rodig, quien, por su lado, también se escribe directamente con Graves: así se dibujan algunas de las líneas que conforman un mapa de relaciones entre importantes escritores, artistas y políticos de la primera mitad del siglo XX.

¿Dónde se encuentra el vínculo, la construcción de un espacio queer en este epistolario? En un primer orden está en la predominancia de la primera persona plural a partir de la cual se construye el discurso epistolar. En las

6 Véase por ejemplo, Díaz Navarrete, Wenceslao, ed. María y los espíritus: diarios y cartas de María Tupper. Santiago: Ediciones UC, 2014. Díaz Navarrete, Wenceslao, ed. Bohemios en París, Epistolario de artistas chilenos en Europa, 1900-1940. Santiago: RiL editores, 2010. 
cartas de 1928 y 1929, Lemetayer se configura frecuentemente desde el nosotras, un gesto que implica la identificación y el reconocimiento de la relación, una relación que tendría sentido para la interlocutora. Una de las primeras cartas de esta colección, fechada el 14 de septiembre de 1928, empieza con "Amiga nuestra" y termina con la firma "Laura y Consuelo". 7 Luego, en el cuerpo de la carta, pasa de primera persona singular al plural en varios momentos. Rodig, por su parte, se hace partícipe de la relación entre Graves y Lemetayer: "Su simpática carta $[. .$.$] la leímos Laura y yo varias veces [\ldots]$ "; pero en otros momentos, particularmente cuando la relación entre Rodig y Lemetayer se empieza a fracturar, desaparece esta conversación de tres mujeres y se transforma en un diálogo exclusivamente entre Lemetayer y Graves. Mientras la relación va bien, los intercambios entre las tres giran en torno a lecturas: "Con Laura hemos empezado a leer de nuevo el Juan Cristóbal de Romain Rolland" (14 de septiembre de 1929); viajes: "Mañana salimos con Laurita para Alemania y creemos alcanzar Berlín" (25 de junio); política: "A los peruanitos no los he visto, parece que hacen campaña privada dirigida por Mariátegui en contra de Víctor" (11 de noviembre de 1928), y los éxitos de Rodig como artista: "Laura ha obtenido la medalla de oro en escultura" (sin fecha). Una de las preguntas preliminares entonces con respecto a las redes queer de este momento es hasta qué punto funcionan como un espacio seguro, de complicidad y de confianza entre sus participantes; donde las cosas que se leen, se piensan y se hacen en pareja se pueden expresar abiertamente. Resulta particularmente interesante en este caso que el uso de la primera persona plural no implica una coincidencia total, un esfuerzo por unificar el discurso, pues el nosotros pensamos aparece en el mismo párrafo con el yo.

La otra tarde nos reunimos en nuestro ricón [sic] de Cachan para tomar una taza de té, Henríquez, Haynes y Bazán, ${ }^{[8]}$ por supuesto que nuestros mejores recuerdos fueron para la amiga ausente. [...] Estuvimos conversando largamente sobre la famosa APRA, conversación que tuvo algo de contradicción porque yo y mi Laura no estamos de acuerdo con ella y la consideramos no una causa noble sino una mera plataforma personal

7 No todas las cartas de esta colección tienen fechas completas. En todos los casos en que existan fechas con año se indicará, en caso contrario se señalará la fecha tal como aparece. Las cartas no están catalogadas individualmente en la colección. Se han agregado las tildes a las palabras, ya que las cartas mecanografiadas no las incluían.

8 Armando Bazán (1902-1962), escritor peruano, comunista, amigo y biógrafo de José Carlos Mariátegui. 
donde magníficas energías se diluyen. [...] A mí estas cosas frívolas de la América del Norte me sacan de quicio. (14 de septiembre de 1928)

Lemetayer y Rodig figuran como parte activa de una comunidad intelectual en el París de entreguerras. Este es un espacio de sociabilidad intelectual mixta que se cruza con las redes queer. Lemetayer y Rodig, por medio de sus cartas, mantienen conectada a Graves con esta comunidad, de la que ella también participa durante sus visitas a París. Este contexto pone de manifiesto la centralidad de la dimensión transnacional de las redes queer. ${ }^{9}$ Como espacios de sociabilidad, intercambio intelectual, apoyo material y afectivo, las redes queer de comienzos de siglo se consolidan y adquieren densidad a partir de los viajes y autoexilios de la mayoría de estas mujeres. Estas redes son el producto de vidas que se resisten a anclarse en el país natal, la familia biológica y el destino que les exigen su clase y su género. Volviendo a la cita anterior, el uso de la primera persona plural en estas cartas es un testimonio de la multidimensionalidad (intelectual, económica, creativa y emocional) de la relación entre estas dos mujeres. Al operar al margen, aunque nunca completamente aisladas de las dinámicas de poder heteronormativo de control patriarcal, su relación representa el potencial amenazador al que se refiere Foucault.

El concepto de queer time, que plantea Halberstam, corresponde a la temporalidad no-normativa de una vida no pautada por las convenciones de reproducción, de herencia o de crianza. Estas convenciones y tiempos están asociados a un ideal de familia burguesa en el contexto de una sociedad que aspira a la respetabilidad "y a las nociones de lo normal de las que esta depende" (Halberstam 4). Si bien Halberstam examina estas ideas en el contexto del postmodernismo, el concepto de tiempo queer constituye un punto de partida para pensar las condiciones de producción, las estrategias de asociatividad y la subjetividad de quienes rechazaron una vida organizada por las demandas de una temporalidad normativa. La libertad para disponer de tiempo y espacio, condición esencial para la creación literaria o artística, como lo plantea Virginia Woolf en A Room of One's Own (1928), solo podía

Organizaciones de mujeres por la paz como WILPF (Women's International League for Peace and Freedom), de la que Graves fue miembro, fueron centros de sociabilidad femenina transnacional e importantes espacios de debate político y social que en muchos casos se enfrentaron a la opinión pública. Véase: Schott, Linda K. Reconstructing Women's Thoughts: the Women's International League for Peace and Freedom Before World War II. Stanford: Stanford University Press, 1997. 
conseguirse a través de independencia económica. La independencia económica permite a la mujer escritora o artista crear alternativas a los patrones de tiempo y espacio que le plantea la sociedad, disponer de su cuerpo, de su tiempo y encontrar espacios donde vivir su vida, su sexualidad y sus relaciones interpersonales con mayor libertad. Teresa de la Parra puede vivir en Europa con su compañera Lydia Cabrera en gran medida gracias al dinero que le hereda otra mujer, Emilia Ibarra; Gabriela Mistral lo logra con los trabajos que consigue y mantiene en virtud de su talento y sus redes; o el caso de Gertrude Stein que vive en París con su compañera Alice B. Toklas, gracias a su herencia y a su creciente fama literaria.

Lemetayer nunca menciona en sus cartas a su exesposo, pero sí comparte con Graves distintos aspectos de su situación económica: "dejé un negocio de agricultura cuyo beneficio nos permitiría vivir holgadamente en París sin preocupaciones pecuniarias. Hoy recibo la mala noticia que en menos de un año nuestro capital se ha perdido" (11 de noviembre de 1928). Más tarde le confiará que depende económicamente de su madre. Su madre quiere que ella regrese a Chile con sus hijas, pero ella se resiste. De todas formas, Lemetayer vive acomodadamente en París y en varias ocasiones ayuda con pasajes y alojamiento a artistas y amigos. Las cartas indican que Lemetayer no mantiene económicamente a Rodig o por lo menos no durante la mayor parte del tiempo en que están juntas. Rodig desde 1929 cuenta con sus propios fondos gracias a una beca del gobierno de Chile: "Buena noticia: El gobierno de mi país acaba de comisionar a Laura para que estudie en Europa tres años más con una renta de 2300 francos mensuales - algo es algo- . Annie querida y yo estoy feliz por este triunfo de mi niña" (25 de mayo de 1929). El financiamiento (en forma de becas, no como misión diplomática) por parte de los estados latinoamericanos para artistas y escritores es un fenómeno moderno que comienza a existir como práctica cultural en las primeras décadas del siglo XIX. Si bien en Chile no es a gran escala, introduce un factor de democratización de la cultura que, como en el caso de Rodig, ofrece autonomía económica a sujetos de clase media que no tenían el privilegio de una herencia o fortuna familiar. Esta beca que obtiene Rodig también significa su participación en una importante red, ya que son 26 los becados (6 son mujeres), varios de los cuales alcanzarán amplio reconocimiento en el mundo del arte, como Camilo Mori, Inés Puyó y Julio Ortiz de Zárate (Zamorano Pérez 192). Las redes —que incluyen a escritores, artistas, críticos, políticos y académicos- ofrecen no solo oportunidades de colaboración e intercambio intelectual y literario, sino también 
apoyo material (trabajos, alojamiento, becas, publicaciones) que resultan fundamentales para la profesionalización de artistas y escritoras. En el caso de mujeres queer que viven fuera de sus países, la red de contactos, amigos y colegas es particularmente significativa ya que entrega acceso a recursos (materiales y simbólicos) que mitigan las pérdidas frecuentemente asociadas a las rupturas con las lógicas heterosexuales y patriarcales de familia, sexualidad y reproducción.

La maternidad se representa de modo ambivalente a lo largo del epistolario. En la breve introducción que acompaña estas cartas, escrita por la misma Anna Melissa Graves, probablemente al momento de compilar sus papeles, describe a Lemetayer como: "A most charming but most utterly irresponsible person. No matter what her duties may be as a daughter or as a mother she is likely to be at the other end of the globe when anyone else would think leaving a child or mother impossible" ("Introducción a las cartas"). Palabras duras de su confidente, que aunque posiblemente mucho más liberal que la mayoría de sus contemporáneas (en tanto escritora, feminista y viajera), critica el "abandono" por parte de una mujer, de sus deberes como madre e hija. Lemetayer no somete su propia voluntad ni su libertad a los espacios ni a los tiempos propios de una mujer de su época y su clase social. Rasgo que comparte con la mayoría de las mujeres que participan de estas redes queer. Aunque las cartas establecen que tuvo a sus hijas internadas en un colegio en Bruselas, lo que sin duda significó mayor libertad para ella en París y podría ser visto negativamente como un "abandono", Lemetayer expresa al mismo tiempo una mirada moderna y feminista con respecto a la educación femenina. La educación de sus hijas significa un gasto considerable, sin embargo, ampliamente justificado según sus propias palabras: "Estoy agradecida con Dios por el alma y el carácter que dio a mis dos hijas - Quiero que ellas queden en Europa, cultivándose, tres años más y que después pasen a Estados Unidos para seguir una carrera noble e útil a la colectividad" (25 de mayo de 1929). No menciona el matrimonio o el regreso a Chile como el destino de sus hijas; su educación no es solo un modo de acrecentar su valor y atractivo matrimonial sino que para Lemetayer es un paso hacia una carrera profesional que contribuya al desarrollo social.

Sin negar lo anterior ni el amor que ella expresa por sus dos hijas en sus cartas, Lemetayer da voz a la frustración del imperativo social que fuerza a la mujer a llenar su vida con sus roles de madre y esposa: 
Mis hijas encantadoras. Margot obtuvo su premio - el único en toda la escuela - de literatura inglesa. Son muy lindas mis hijas, Annie, quiero decir en alma e inteligencia... Ah! Si yo fuese SOLAMENTE MADRE, pero no puedo [...] Queda un vacío tan enorme dentro de mi corazón que por ley HUMANA yo necesito llenarlo [...] ¡Y lo lleno tan mal! (mayúsculas y puntuación originales, 15 de julio, sin año)

Lemetayer es capaz de verbalizar la crisis de sentido que el imperativo de la maternidad le provoca, que la lleva a cuestionar su subjetividad y los deseos - afectivos, sexuales y creativos - que no le permiten conformarse con este rol. La mujer, que en las primeras décadas del siglo XX vive una vida queer, no lo hace como una decisión limpia y placenteramente revolucionaria. Desafiar los roles de género tradicionales, los tiempos y los espacios pauteados por la sociedad patriarcal tiene un costo enorme, es un proceso marcado por la ansiedad y la culpa.

La relación de Lemetayer con Rodig incluye también a sus hijas. Rodig apoya a Lemetayer con el cuidado de sus hijas, por ejemplo, cuando esta última tiene que viajar a Chile, le cuenta a Graves que dejará a Margot a cargo de Laura: "Laura me pide enviarle, junto con su cariño, esas dos fotografías suyas. En estos momentos ella y yo estamos preparando muy apuradas nuestros viajes: el mío a Chile y el de ella junto con mi hijita Margot a la Alta Savoya francesa que queda justamente sobre Ginebra" (29 de noviembre de 1928). Compartir estas responsabilidades plantea un modelo queer de familia que, fuera del esquema heterosexual, crea otro modo de división de la labor doméstica, un modelo que también se observa en esta misma época en la relación entre Gabriela Mistral, Palma Guillén y el hijo adoptivo de la primera, Yin-Yin.

El matrimonio y las relaciones románticas aparecen como un tema de discusión que circula entre estas mujeres. En la misma carta en que Lemetayer manifiesta su insatisfacción con el imperativo de la maternidad, comenta las ideas acerca del matrimonio de compañeros que el juez Ben Lindsay desarrolla en su libro The Companionate Marriage (1927):

Gracias muchas, mi buena amiga por sus libros. Estoy leyendo el del Juez Ben Lindsey. Es lo más humano, noble y verdadero que se ha dicho PERO en el estado de cosas actual, de nuestros códigos y de nuestros regímenes, aquello tan lindo y sentido que el Juez Lindsey dice, don't work out. Él es un cristiano, un poeta sensible y sincero [...] PERO tan LINDAS COSAS no pueden existir. La montaña de sucios prejuicios es tan enorme que quien quiera destruirla es destruido por ella. (15 de julio, sin año; mayúsculas y puntuación originales) 
El libro del juez Lindsey propone un matrimonio que permita a la pareja conocerse y probar su compatibilidad y que, con la condición de que no haya hijos, puede disolverse fácilmente sin pago de pensiones. Su planteamiento, que evidentemente causó gran polémica en Estados Unidos, sitúa la compatibilidad emocional y sexual de la pareja en el centro del matrimonio, cuestionando además el vínculo entre matrimonio y procreación. Lemetayer concuerda con la visión de Lindsey, pero es tajante en cuanto a la capacidad de la sociedad de incorporar estas ideas. Los códigos, los regímenes y los prejuicios son para Lemetayer un muro imposible de traspasar, y quien quiera intentarlo es destruido. Aparece aquí nuevamente la marca de la ansiedad y la desesperanza presente en muchas de estas mujeres, quienes, conscientes de los riesgos de no someterse a las convenciones, viven en una constante batalla para no ser destruidas: desheredadas, alejadas de sus hijos, imposibilitadas de trabajar, aisladas socialmente o encerradas.

Es interesante examinar estas relaciones y las redes queer en sus vínculos con el desarrollo creativo e intelectual de estas mujeres y particularmente con respecto a sus procesos de profesionalización. ¿Hasta qué punto estas relaciones favorecen sus estrategias de profesionalización y de desarrollo creativo, frente a un establishment literario e intelectual que las margina?

En un primer nivel se destaca la dimensión intelectual en la relación de Rodig y Lemetayer. Consuelo le lee a Laura una novela mientras ella pinta, y luego la discuten. Asisten juntas a reuniones donde se discute de política y de arte. Reciben reconocimiento de otros como una pareja activa en el campo cultural del momento. Así queda en evidencia en la única carta encontrada hasta el momento - fuera de este epistolario - en la que aparecen las dos mencionadas. Es una carta que César Alfredo Miró Quesada, escritor y compositor peruano, amigo de Vallejo en París, le escribe a Mariátegui:

Consuelo Lemetayer y Laura Rodig me encargan un saludo muy cariñoso para U. Le adjunto un artículo sobre esta última y algunas reproducciones de sus obras. Me pide Consuelo que le diga que ha recibido su carta, así como la que va dirigida a Rabines. Le contestará en estos días. Me dice también que ha conseguido algunas suscripciones para Chile que le enviará. (París, 27 de septiembre de 1929)

Rodig es una artista activa profesionalmente, trabaja, participa en exposiciones y estudia en la Academia de André Lhote. Lemetayer está conectada con escritores latinoamericanos y franceses, y no parece tener un trabajo remunerado; sin embargo, apoya a la revista Amauta en París y opta 
por dedicarse a facilitar la carrera de Rodig: "Ahora es mi niña grande quien me necesita, es el calor de mi ternura y mi comprensión lo que hará su obra más fácil; ya una vez que ella llegue a la meta y tal vez no me necesite, iré yo donde Ud. Annie dear" (14 de septiembre de 1928). El apoyo que Lemetayer le ofrece a Rodig es emocional pero también es logístico y estratégico. Hay una dimensión maternal en esta relación, aunque no es posible determinar hasta qué punto es solo nominal - un modo de nombrar la relación-, para codificarla en términos socialmente válidos o si, efectivamente, Lemetayer actúa como una madre de la cual Rodig depende, aunque no parece ser el caso. ${ }^{10}$ En cuanto a la participación de Lemetayer en la carrera de Rodig, hay varios ejemplos a lo largo del epistolario de cómo la primera trabaja en la difusión de la obra de la segunda, manda fotografías de su obra y recortes de prensa a amigos y colegas, gestiona invitaciones y la apoya en sus momentos de trabajo y descanso.

Lemetayer, que dominaba el inglés y el francés, sin duda facilitó el contacto de Rodig con figuras claves de la cultura en Europa y Estados Unidos. Al menos con Graves, funciona así, ya que Lemetayer muchas veces actúa como puente entre ellas. Lemetayer aparece preocupada por promover la carrera de Rodig y conseguir oportunidades para ella. Así lo expresa en esta carta de agosto de 1930: "Le mando ese Monde donde Barbusse ha ilustrado su novela inédita con un dibujo original de Laura. Ignoro por qué el nombre de Laura no figura. ${ }_{\mathrm{C}}$ Ha sido olvido simple? Esto me servirá para hacer introducir a Laura más profundamente en Monde porque considero ese contacto muy importante para ella" (13 de agosto de 1930). No es posible saber si Graves contribuyó a la publicación del dibujo de Laura en la revista Monde, pero lo que sí es un hecho es que la norteamericana mantenía una correspondencia con Henri Barbusse, su fundador y director. Lo que queda claro es que Lemetayer está comprometida con el éxito de Rodig como artista y para esto se preocupa por la visibilidad de su obra y por establecer contactos estratégicos.

Un último punto de análisis de este epistolario es la dimensión emocional que marca esta relación. Las cartas de Lemetayer dan cuenta de los distintos sentimientos que le provoca su relación con Rodig. Si bien estas cartas constituyen un espacio seguro, donde Lemetayer le confía a Graves sus emociones y ansiedades, sus palabras están atravesadas por una ambivalencia

10 Esta codificación maternal de las relaciones románticas entre mujeres también se observa en el epistolario entre Mistral y Doris Dana. Véase: Mistral, Gabriela. Niña errante: cartas a Doris Dana. Ed. Pedro Pablo Zegers. Santiago: Lumen, 2010. 
que revela pero al mismo tiempo oculta. La teoría feminista en la literatura ha establecido la codificación como una estrategia textual clásica para inscribir un deseo homosexual que no se atreve a manifestarse abiertamente, "the love that dare not speak its name".

Hay entre Graves y Lemetayer una comprensión que se basa en parte en aceptar los sentimientos de la otra y en saber entenderlos: "Cuénteme su vida, Ud. sabe cuánto la quiero y la comprendo. Laura queda en París para hacer su exposición en enero. Tuve mucha pena de dejarla" (Rio de Janeiro, 7 de noviembre de 1929; énfasis original). Lemetayer también sirve de confidente de Graves con sus propios problemas (que no han formado parte de este análisis) y parece ser que solo tienen que contarse un poco para que la otra entienda la dimensión de sus emociones o miedos. En el epistolario hay tres momentos de la relación entre las chilenas. Un primer momento en que Lemetayer expresa felicidad y entusiasmo, y se refiere frecuentemente a "mi Laura", "mi niña grande", concentrando la conversación en los éxitos de Laura, los viajes, libros y proyectos. En un segundo momento emergen señales de crisis que Lemetayer enfrenta con la ayuda de los consejos de Graves. En abril de 1930 hay una primera señal de alejamiento de la escultora: "Laura ahora ha tomado un gran taller para trabajar libremente. Aquí el apartamento resultaba chico, mucho más viniendo mis chicas a pasar sus vacaciones" (14 de abril de 1930). Más adelante le cuenta algunas dificultades en la relación y que aunque Laura ha buscado un taller fuera de la casa, hay armonía entre ellas: "Laura bien - por fortuna la armonía reina y dulcemente se sigue el camino verdadero" (9 de octubre), "Con Laura estamos muy felices - los consejos suyos y que seguí, me hicieron bien- ojalá que esta comunión y alegría sea larga" (14 de septiembre de 1930). ¿Qué constituye este "camino verdadero"? ¿Es este camino una relación de compañerismo y colaboración como la que plantea el juez Lindsay en su libro de matrimonio de compañeros? ¿Qué espacios y temporalidades permiten a Consuelo seguir el camino verdadero de su relación con Laura? No es posible tener una respuesta definitiva a todas estas preguntas, pero sí continuar esta investigación para determinar hasta qué punto el autoexilio, la participación en redes de mujeres queer, la resistencia a los modelos tradicionales de familia son condiciones necesarias para vivir este "camino verdadero".

El final de la relación aparece en las últimas cartas. Su manifestación más tajante es: "Laura no vive ya conmigo $[\ldots]$ ¡Necesito tanto verla, tanto, mi amiga única! Estos últimos meses han sido muy duros para mí, y aunque no quiero confesarlo, la partida de Laura me ha muerto" (25 de abril de 1931). El fin de la relación provoca en Lemetayer una pérdida de sentido de la vida: 
"Laura [...] Todo mi coraje está en este punto. TODO mi esfuerzo para que mi pobre vida de tantas luchas no se hunda en el vacío" (Martes). Sin embargo, a pesar de la desilusión, Lemetayer expresa en esta misma carta la idea de que su vida tiene muchas dimensiones, de modo muy similar a como lo planteaba con respecto a la maternidad, y que ella puede renacer si se conecta con esas otras necesidades: "He comprendido muchas cosas que me han ido hondo al corazón dañándolo enormemente pero también al lado de eso tan FEO veo otras actividades, otras necesidades a las cuales mi vida es absolutamente NECESARIA" (Martes; mayúsculas originales). Este discurso acerca del amor como una más, entre otras, necesidades de un sujeto, es un mensaje que contraviene el imperativo social del amor romántico heterosexual en la mujer, como un evento central y definitivo en la vida, que al perderse, la deja sin identidad ni sentido. Lemetayer sufre la pérdida de su compañera, sin embargo, tal vez porque ha organizado su vida en términos muy diferentes a la mayoría de las mujeres de su clase, se refugia en su red de amigos y amigas, como Graves, y en un sentido de propósito que ella le da a su vida y que va más allá de su rol como madre o amante.

Lamentablemente, poco sabemos del final de la vida de esta mujer. Este epistolario inédito la ha rescatado del olvido para revelar una etapa de su vida, una vida queer, que permite continuar el trabajo de recuperación y reevaluación que, desde el feminismo y los estudios de género, se propone construir una historia cultural más diversa y compleja. Esta historia es solo una pieza en la red de mujeres latinoamericanas queer de la primera mitad del siglo XX. La reconstrucción de esta red es un paso clave para analizar las condiciones de producción cultural, no solo como simples condiciones, sino como algo que permea y determina ideologías, poéticas, proyectos intelectuales y prácticas políticas. En un periodo en que las relaciones heterosexuales y la vida de las mujeres bajo un imperativo patriarcal y heterosexual no otorga, por lo general, el apoyo y el espacio para la expresión de una subjetividad personal y para el desarrollo de proyectos creativos e intelectuales, resulta productivo observar las dinámicas que se dan en estas alianzas no normativas y en las redes queer. Y por último, es necesario preguntarse cuáles fueron los costos profesionales y personales de una vida queer. Porque además de pensar acerca de las relativas ventajas que estos modos alternativos de asociatividad ofrecían, también es fundamental visibilizar la discriminación y la violencia que operaban sobre las mujeres que resistían la normatividad heterosexual, reproductiva y de dependencia económica, y el impacto de esta violencia en el campo cultural. 


\section{Obras citadas}

Foucault, Michel. "Friendship as a way of life". Ethics: Subjectivity and Truth. Paul Rabinow, vol. 1 of The Essential Works of Foucault. New York: New Press, 1997. Impreso.

Graves, Melissa. "Introducción a las cartas". Swarthmore College Peace Collection, Anna Melissa Graves Papers, 1919-1953. Impreso.

Halberstam, Judith. In a Queer Time and Place: Transgender Bodies, Subcultural Lives. New York: New York University Press, 2005. Impreso.

Lemetayer, Consuelo. "Cartas a Anna Melissa Graves. 1928-1931". Swarthmore College Peace Collection, Anna Melissa Graves Papers, 1919-1953. Manuscrito. Miró Quesada, César Alfredo. "Carta a Mariategui, 27 de septiembre de 1929". Fosé Carlos Mariategui. Correspondencia Tomos I y II. www.mariategui.eu. Web.

Peña Castro, Susana. "Re: Laura Rodig". Recibido por Claudia Cabello Hutt, 18 de mayo de 2017.

Taylor, Martin C. Gabriela Mistral's Struggle with God and Man. A Biographical and Critical Study of the Chilean Poet. Jefferson, NC: McFarland, 2012. Impreso.

Zamorano Pérez, Pedro Emilio. "Educación artística en Chile.

Fernando Álvarez de Sotomayor, Juan Francisco González y

Pablo Burchard". Atenea 495 (2007): 185-211. Impreso. 